\title{
AN ANALYSIS OF LOGISTIC GROWTH MODEL WITH IMMIGRATION FUNCTION IN FUZZY ENVIRONMENT
}

\section{HARISH CHANDRA BHANDARI ${ }^{\mathrm{a} 1}$ AND KANHAIYA JHA ${ }^{\mathrm{b}}$}

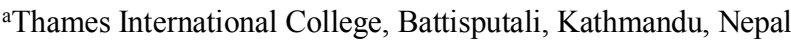

${ }^{b}$ School of Science, Kathmandu University, Dhulikhel, Nepal

\begin{abstract}
In this paper, we discuss the stability analysis of logistic growth model with immigration function in fuzzy environment. The notion of generalized Hukuhara (gH) differentiability is used for the analysis when the immigration function is proportional to the population and some new results have been established with numerical verification.
\end{abstract}

KEYWORDS: Fuzzy Sets, Stability Theory, Fuzzy Differential Equations, gH-Differentiability

Applied analysis play an important role to model real natural phenomena; like in the field of economics, biomathematics, science and engineering. But in the modern era, it is believed that the information about the physical phenomena may be uncertain. The concept of fuzzy set theory is a powerful tool to overcome this impreciseness. The concept of fuzzy set theory and differential equations separately lead to focus their work towards the new concept of the Fuzzy Differential Equations (FDEs). In recent year, the research on FDEs has been growing rapidly. L.A. Zadeh introduced the term fuzzy for the first time in 1965 (Zadeh, 1965) and had given lots of examples where the nature of uncertainty in behavior of given system possesses a fuzzy phenomenon. In 1972, the fuzzy derivative was first conceptualized by (Chang and Zadeh, 1972) whereas the fuzzy derivative was first introduced by (Kandel and Byatt, 1978).

Based on Zadeh's extension principle, Dubois and Prade in 1982, presented the elementary fuzzy calculus. Also, (Kaleva, 1987) \& (Kaleva, 1990) and (Seikkala, 1987), studied the FDEs and fuzzy initial value problems. (Bede, 2008) presented two characterization theorem for the solution of FDEs which translate FDEs into crisp ordinary differential equations. In 2012, the new concept of generalized derivative for fuzzy mappings and FDEs were discussed by (Platnikov and Skripnik, 2012). Also, (Mondal et.al, 2013) solved the first order linear homogeneous ordinary differential equation in fuzzy environment. Two different types of solutions of fuzzy functional integrodifferential equations were studied by (Hoa and Phu, 2014). Recently, (Paul et al., 2016) described the solution of fuzzy quota harvesting model in fuzzy environment. The logistic growth model proposed by Pierre-Francois Verhulst in 1838 is as follows:

$$
\frac{d P}{d t}=r P\left(1-\frac{P}{K}\right)
$$

where $\mathrm{P}(\mathrm{t})$ represents the population size at time $\mathrm{t}, \mathrm{r}$ is the intrinsic growth rate and $\mathrm{K}$ is the carrying capacity. Also, if $\mathrm{f}(\mathrm{P})$ is the immigration function then, the modified logistic growth equation discussed by (Kumar and Lal, 2017) is

$$
\frac{d P}{d t}=r P\left(1-\frac{P}{K}\right)+f(P)
$$

They have discussed the stability analysis of the model equation (2) with numerical verifications for $f(P)$ proportional to $P^{\alpha}$ (for $\alpha=1,2,3$ ) in a crisp setting.

Now, we have extended this model equation (2) for the stability analysis of the equilibrium points for fuzzy parameters when the immigration function $f(P)$ is proportional to $P$. The purpose of this paper is to establish some new results for the stability analysis of logistic growth model with immigration function in fuzzy environment by the concept of (Paul et al.,, 2016) in the model equation (2) established by (Kumar and Lal, 2017).

\section{PRELIMINARY CONCEPTS}

Westart with the following definitions:

Definition 2.1 (Tapaswini and Chakraverty, 2013) A fuzzy number is a function such as $u: R \rightarrow(0,1)$ satisfying the following properties:

1. $\mathrm{u}$ is normal, i.e $\exists x_{0} \in R$ with $u\left(x_{0}\right)=1$.

2. $\mathrm{u}$ is a convex fuzzy set i.e $u(\lambda x+(1-\lambda) y) \geq$ $\min \{u(x), u(y)\} x, y \in R, \lambda \in(0,1)$ 
3. $\mathrm{u}$ is upper semi-continuous on $\mathrm{R}$.

4. $\overline{\{x \in R: u(x)>0\}}$ is compact where $\bar{A}$ denotes the closure of $A$.

Definition 2.2 (Tapaswini and Chakraverty, 2013) A fuzzy number $\tilde{A}$ is said to be triangular if its membership function $\mu_{\tilde{\mathrm{A}}}$ is given by

$\mu_{\tilde{\mathrm{A}}}(x)=\left\{\begin{array}{ccc}\frac{x-a}{b-a} & \text { if } & a \leq x \leq b \\ \frac{c-x}{c-b} & \text { if } & b \leq x \leq c \\ 0 & \text { otherwise }\end{array}\right.$

Definition 2.3 (Hooshangian, 2016) A fuzzy number $\widetilde{\boldsymbol{u}}$ in parametric form is a pair $(\underline{u}, \bar{u})$ of functions $\underline{u}(\alpha)$, $\bar{u}(\alpha), \quad 0 \leq \alpha \leq 1$ which satisfy the following conditions:

a. $u(\alpha)$ is bounded non-decreasing left continuous function in $(0,1)$, and right continuous at 0 .

b. $u(\alpha)$ is bounded non-increasing left continuous function in $(0,1)$, and right continuous at 0 .

c. $u(\alpha) \leq u(\alpha), 0 \leq \alpha \leq 1$.

Definition 2.4 (Takata et.al, 2015) The generalized Hukuhara difference (gH-difference) of two fuzzy numbers

$\tilde{u}, \tilde{v} \in F(R)$ is defined as follows:

$\tilde{u} \ominus_{g H} \tilde{v}=\widetilde{w}$ is equivalent to $\left\{\begin{array}{lr}(i) \tilde{u}= & \widetilde{v} \oplus \widetilde{w} \\ (i i) \widetilde{v}=\tilde{u} \oplus(-1) \widetilde{w}\end{array}\right.$.

In terms of $\alpha$-cut, $\tilde{u} \ominus_{g H} \tilde{v}=\widetilde{w}$ is equivalent to $(\widetilde{w})_{\alpha}=$ $[\underline{w}(\alpha), \bar{w}(\alpha)]$ where,

$$
\underline{w}(\alpha)=\min \{\underline{u}(\alpha)-\underline{u}(\alpha), \quad \bar{u}(\alpha)-\bar{v}(\alpha)\}
$$

and

$$
\bar{w}(\alpha)=\min \{\underline{u}(\alpha)-\underline{u}(\alpha), \quad \bar{u}(\alpha)-\bar{v}(\alpha)\} .
$$

Definition 2.5 (Mondal et.al, 2016) The generalized Hukuhara derivative of a fuzzy valued function

$f:(a, b) \rightarrow F(R)$ at point $t_{0}$ is defined as

$$
f^{\prime}\left(t_{0}\right)=h \stackrel{\lim }{\rightarrow} \frac{f\left(t_{0}+h\right) \ominus_{g H} f\left(t_{0}\right)}{h}
$$

If $f^{\prime}\left(t_{0}\right) \in F(R)$,satisfying the definition of fuzzy set, then $\mathrm{f}$ is generalized Hukuhara differentiable at $t_{0}$. Also, $f(t)$ is (i) $-g H$ differentiable at $t_{0}$ if

$$
\left(f^{\prime}\left(t_{0}\right)\right)_{\alpha}=\left(f^{\prime}\left(t_{0}, \alpha\right), \overline{f^{\prime}}\left(t_{0}, \alpha\right)\right)
$$

and $f(t)$ is $(i i)-g H$ differentiable at $t_{0}$ if

$$
\left(f^{\prime}\left(t_{0}\right)\right)_{\alpha}=\left(\overline{f^{\prime}}\left(t_{0}, \alpha\right), \underline{f^{\prime}}\left(t_{0}, \alpha\right),\right)
$$

Definition 2.6 (Mondal et.al, 2016) The solution of the fuzzy differential equation of the form $(\underline{x}(t, \alpha), \bar{x}(t, \alpha))$, is called strong solution if

$$
\frac{\partial \underline{x}(t, \alpha)}{\partial t}>0, \frac{\partial \bar{x}(t, \alpha)}{\partial t}<0 \forall \alpha \in[0,1], \underline{x}(t, \alpha) \leq \bar{x}(t, \alpha)
$$

otherwise it is weak solution.

\section{MAIN RESULTS}

When we consider the logistic growth equation for immigration function $f(P)=c P$, the modified logistic growth equation from $(2)$ is

$$
\frac{d P}{d t}=r P\left(1-\frac{P}{K}\right)+c P
$$

We have discussed for the following three possible cases when:

a) Initial population is a fuzzy number,

b) Natural birth rate and immigration coefficient are fuzzy numbers, and

c) Initial population as well as natural birth rate and immigration coefficient are fuzzy numbers.

Before discussing about the stability of the equation (3) we need the following new theorems:

Theorem 3.1 All the equilibrium points of the system of equations

$$
\left\{\begin{array}{l}
\frac{d x}{d t}=p x\left(1-\frac{y}{K}\right)+q x \\
\frac{d y}{d t}=r x\left(1-\frac{x}{K}\right)+q y
\end{array}\right.
$$

where $p, q, r, s$ and $K$ are all positive constants, are unstable.

Proof: $\operatorname{If}\left(x^{*}, y^{*}\right)$ is an equilibrium point of the system (4), then $\frac{d x^{*}}{d t}=0$ and $\frac{d y^{*}}{d t}=0$.

On simplification, we get $(0,0)$ and $E_{1}=(K(1+$ $\left.\left.\frac{s}{r}\right), K\left(1+\frac{p}{q}\right)\right)$ as the equilibrium points of the system (4). So the Jacobian matrix is 
$J_{1}=\left[\begin{array}{cc}p\left(1-\frac{y}{K}\right)+q & -\frac{p x}{K} \\ -\frac{r y}{K} & r\left(1-\frac{x}{K}\right)+s\end{array}\right]$.

Now, the Jacobian matrix at point $(0,0)$ is

$$
J_{1}(0,0)=\left[\begin{array}{cc}
p+q & 0 \\
0 & r+s
\end{array}\right] .
$$

The characteristic equation of $J_{1}(0,0)$ is

$$
\lambda^{2}-a \lambda+b=0
$$

where,

$$
a=\operatorname{Tr}\left(J_{1}(0,0)\right)=p+q+r+s
$$

and

$$
b=\operatorname{Det}\left(J_{1}(0,0)\right)=(p+q)(r+s) .
$$

If $a<0$ and $b>0$, then by Routh-Hurwitz criteria, we find that the system is stable but here $a=p+$ $q+r+s>0$, so the equilibrium point $(0,0)$ is unstable.

Now, at equilibrium point $E_{1}$, the Jacobian matrix is

$$
J_{1}\left(E_{1}\right)=\left[\begin{array}{cc}
0 & -p\left(1+\frac{s}{r}\right) \\
-r\left(1+\frac{q}{p}\right) & 0
\end{array}\right] .
$$

Again, the characteristic equation of $J_{1}\left(E_{1}\right)$ is given by

$$
\lambda^{2}-a_{1} \lambda+b_{1}=0
$$

where,

$a_{1}=\operatorname{Tr}\left(J_{1}\left(E_{1}\right)\right)=0 \quad$ and $\quad b_{1}=\operatorname{Det}\left(J_{1}\left(E_{1}\right)\right)=$ $-p r\left(1+\frac{s}{r}\right)\left(1+\frac{q}{p}\right)$.

Therefore, by Routh-Hurwitz criteria, we see that the equilibrium point $E_{1}$ is unstable.

If we interchange the variables $\mathrm{x}$ and $\mathrm{y}$ in the left hand side of the system of differential equations in the Theorem 3.1, we have the following result.

Theorem 3.2 Consider a system of differential equations

$$
\left\{\begin{array}{l}
\frac{d y}{d t}=p x\left(1-\frac{y}{K}\right)+q x \\
\frac{d x}{d t}=r x\left(1-\frac{x}{K}\right)+q y
\end{array}\right.
$$

where $p, q, r, s$ and $K$ are all positive constants. Then, the trivial equilibrium point is unstable and nontrivial equilibrium point is unconditionally stable.

The proof is as similar as in Theorem 3.1.

We consider the model equation when the initial population is a fuzzy number and the solution is as follows:

If $\widetilde{P}(0)=\widetilde{P}_{0}$ is a triangular fuzzy number. Then, the following two sub cases arise:

Case I: When $\tilde{P}(t)$ is $(i)-g H$ differentiable and its $\alpha$-cut is $(\tilde{P}(t))_{\alpha}=(\underline{P}(t), \bar{P}(t))$.

Then, the model equation (3) becomes

$$
\left\{\begin{array}{l}
\frac{d \underline{P}}{d t}=r \underline{P}\left(1-\frac{\bar{P}}{K}\right)+c \underline{P} \\
\frac{d \bar{P}}{d t}=r \bar{P}\left(1-\frac{P}{K}\right)+c \bar{P}
\end{array}\right.
$$

Now, by using Theorem 3.1, we can claim that all the equilibrium points of the system (6) are unstable.

Case II: When $\tilde{P}(t)$ is $(i i)-g H$ differentiable.

Then, the model equation (3) becomes

$$
\left\{\begin{array}{l}
\frac{d \bar{P}}{d t}=r \underline{P}\left(1-\frac{\bar{P}}{K}\right)+c \underline{P} \\
\frac{d \underline{P}}{d t}=r \bar{P}\left(1-\frac{P}{K}\right)+c \bar{P}
\end{array}\right.
$$

Using the Theorem 3.2, we can claim that the trivial equilibrium point $(0,0)$ of the system is unstable and non-trivial equilibrium point $\left(K\left(1+\frac{c}{r}\right), K\left(1+\frac{c}{r}\right)\right)$ is unconditionally stable.

The two figures (1) represent the graphs when $\tilde{P}(t)$ is $(i i)-g H$ differentiable for $r=0.1, c=0.05, K=$ 100 and the fuzzy initial value $\tilde{P}(0)=(45,55,65)$ in which crisp solution lies between the fuzzy solutions and fuzzy solutions are closure to crisp solution when $\alpha$ is increased from 0.1 to 0.8 . Therefore, the fuzzy solution is the strong fuzzy solution. 
BHANDARI AND JHA: AN ANALYSIS OF LOGISTIC GROWTH MODEL WITH IMMIGRATION FUNCTION IN...
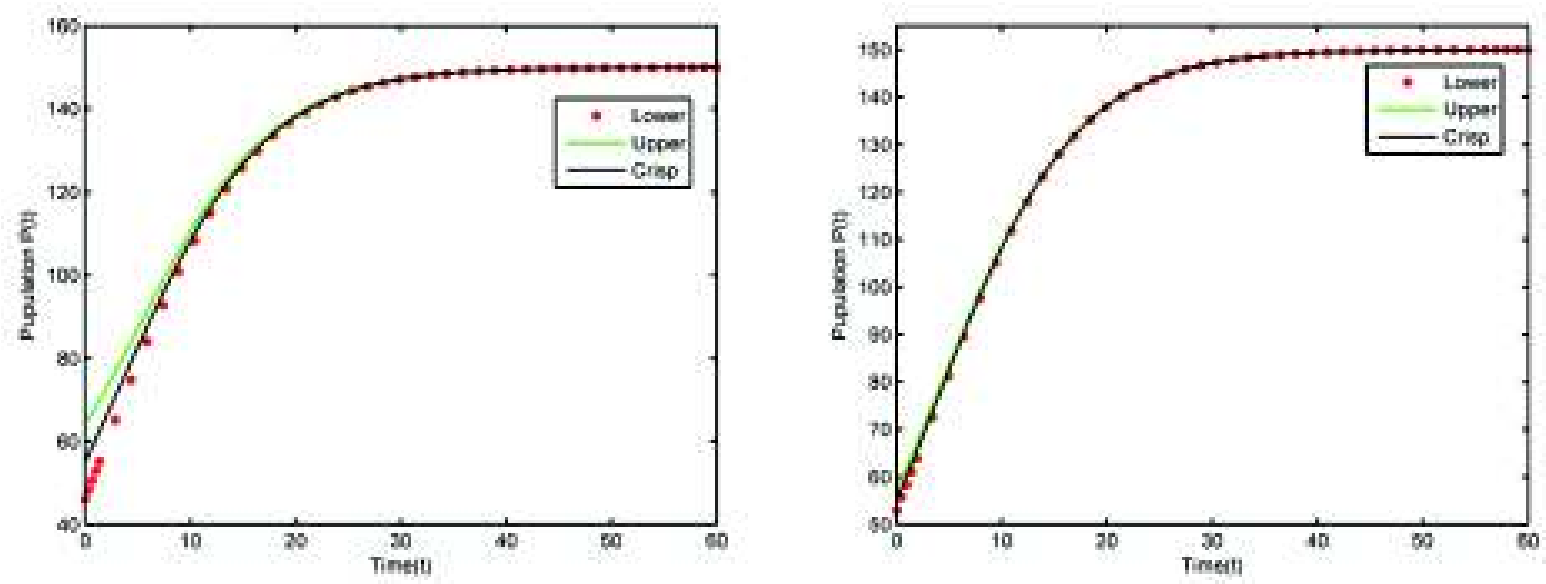

Figure 1: Graph of $(i i)-g H$ differentiable LGM with $f(P)=c P$ for fuzzy initial value

Now, we consider the growth rate and immigration rates as fuzzy numbers which are given by following cut sets $\tilde{r}=[\underline{r}, \bar{r}]$ and $\tilde{c}=[\underline{c}, \bar{c}]$. Then, we have the following two cases:

Case I: When $\tilde{P}(t)$ is $(i)-g H$ differentiable.

Then, the model equation (3) implies the following

$$
\left\{\begin{array}{l}
\frac{d \underline{P}}{d t}=\underline{r P}\left(1-\frac{\bar{P}}{K}\right)+\underline{c P} \\
\frac{d \bar{P}}{d t}=\bar{r} \bar{P}\left(1-\frac{P}{K}\right)+\bar{c} \bar{P}
\end{array}\right.
$$

So, the system of differential equations is unstable for every equilibrium points using Theorem3.1.

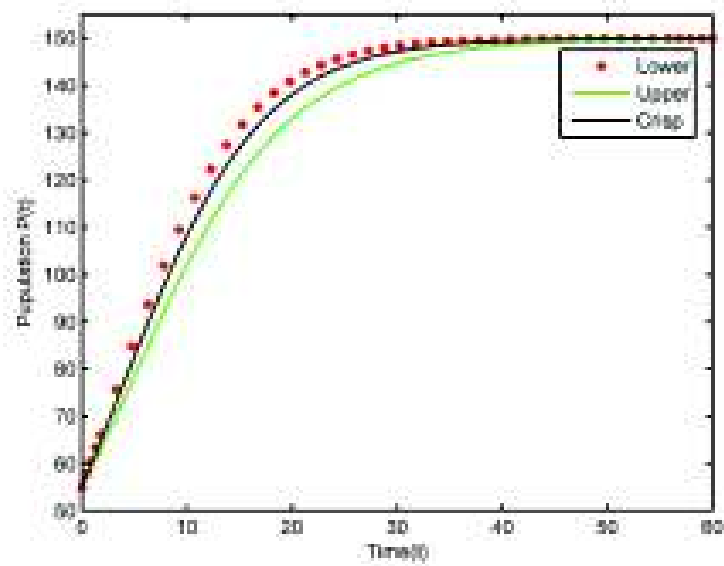

Case II: When $\tilde{P}(t)$ is $(i i)-g H$ differentiable. Then, the model equation (3) is transformed into

$$
\left\{\begin{array}{l}
\frac{d \bar{P}}{d t}=\underline{r P}\left(1-\frac{\bar{P}}{K}\right)+\underline{c P} \\
\frac{d P}{d t}=\bar{r} \bar{P}\left(1-\frac{P}{K}\right)+\bar{c} \bar{P}
\end{array}\right.
$$

So, the system has the trivial equilibrium point $(0,0)$ which is unstable and non-trivial equilibrium point $\left(K\left(1+\frac{\bar{c}}{\bar{r}}\right), K\left(1+\frac{c}{\underline{r}}\right)\right)$ which is unconditionally stable from the concept of Theorem 3.2.

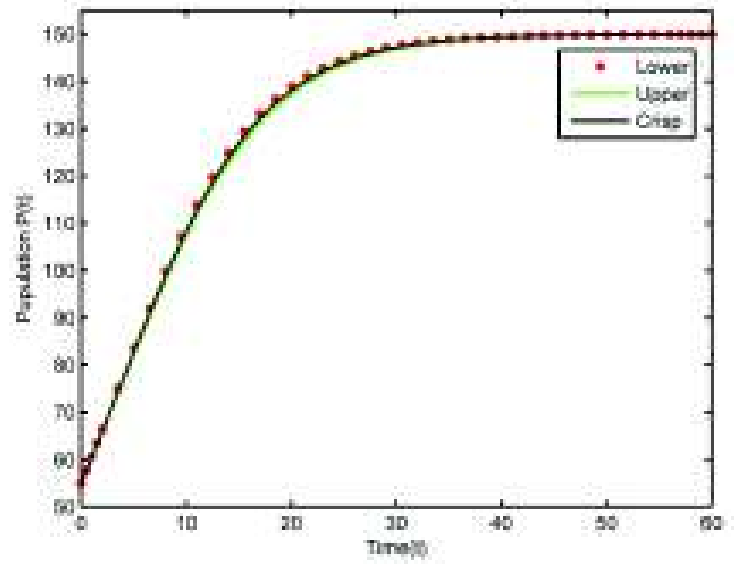

Figure 2: Graph of (ii)-gH differentiable LGM with $f(P)=c P$ for fuzzy intrinsic growth rate

The two figures (2) represent the graphs when $\tilde{P}$ is (ii) $-g H \quad$ differentiable for $\tilde{r}=(0.08,0.1,0.12), \tilde{c}=$
$(0.04,0.05,0.06), K=100$ and the fuzzy initial value $P(0)=55$ in which crisp solution lies be- tween the 
BHANDARI AND JHA: AN ANALYSIS OF LOGISTIC GROWTH MODEL WITH IMMIGRATION FUNCTION IN...

fuzzy solutions and fuzzy solutions are closure to crisp solution when $\alpha$ is increased from 0 to 0.5 . Therefore, the fuzzy solution is justified but it is not strong fuzzy solution. The parameter chosen satisfy the above condition for the stability and trajectories above gives the verification for the stability.

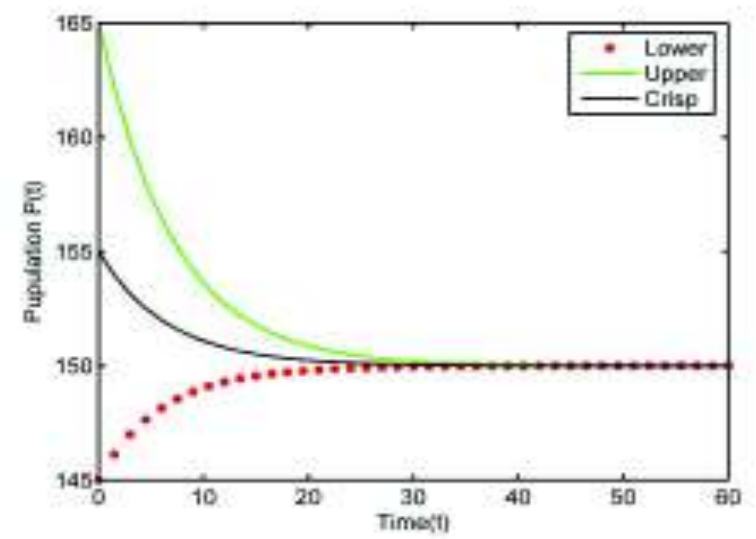

Now, we consider the case when the initial population as well as growth rate and immigration rates are fuzzy numbers so that the stability condition in this section is exactly same as previous in which intrinsic growth rate and immigration constant are fuzzy numbers. Therefore, only numerical verification is presented here.

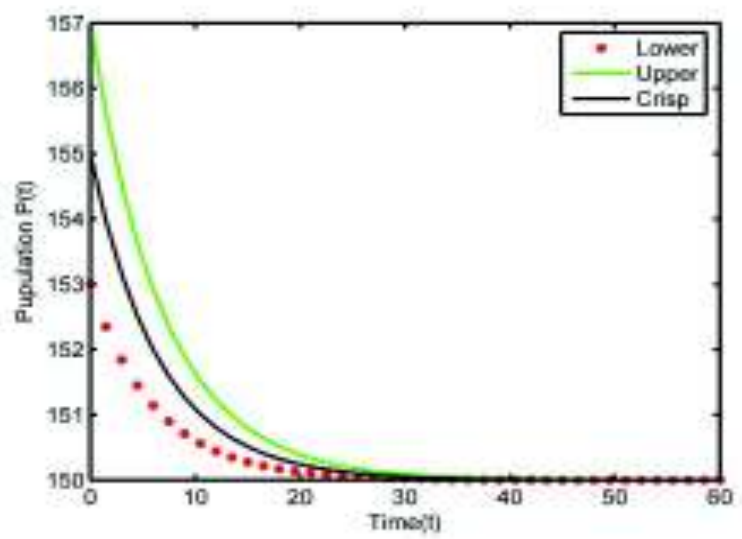

Figure 3: Graph of (ii)-gH differentiable LGM with $f(P)=c P$ for fuzzy initial value as well as fuzzy intrinsic growth rates

The two figures (3) represent the graph of the hypothetical data when $\tilde{P}$ is $(i i)-g H$ differentiable for $\tilde{r}=$ $(0.096,0.1,0.104), \tilde{c}=(0.048,0.05,0.052), K=$ 100 and the fuzzy initial value $\widetilde{P}(0)=(145,155,165)$ in which crisp solution lies between the fuzzy solutions and fuzzy solutions are closure to crisp solution when $\alpha$ is increased from 0 to 0.5 . Therefore, the fuzzy solution is justified but it is not strong fuzzy solution. The parameter chosen satisfy the above condition for the stability and trajectories above gives the verification for the stability.

\section{CONCLUSION}

Some new results have been established to show the stability of logistic growth model with immigration function in $g H$-differentiability and successfully applied. The results so obtained is justifiable analytically and verified graphically in fuzzy environment. Our results expand the results of (Kumar and Lal, 2017) on logistic growth model with immigration function that is proportional into fuzzy version.

\section{ACKNOWLEDGMENT}

The authors would like to acknowledge University Grants Commission (UGC), Nepal for providing financial support under Science and Technology stream.

\section{REFERENCES}

Bede B., 2008. Note on "Numerical solution of fuzzy differential equations by predictor corrector method". Information Sciences, 178: 1917-1922.

Chang S.L. and Zadeh L.A., 1972. On fuzzy mapping and control. IEEE Transaction on Systems, Man and Cybernetics, 2(1): 30-34.

Hoa N.V. and Phu N.D., 2014. Fuzzy functional integrodifferential equations under generalized $\mathrm{H}$ differentiability. Journal of Intelligent \& Fuzzy System, 26(4): 2073-2085.

Hooshangian L., 2016. Analytic Solution of second order differential equations under H-derivation. Turkish Journal of Fuzzy Systems, 7(1): 55-67.

Kaleva O., 1987. Fuzzy differential equations. Fuzzy Sets and Systems, 24(3): 301-317.

Kaleva O., 1990. The Cauchy problem for fuzzy differential equations. Fuzzy Sets and Systems, 35(3): 389396. 
BHANDARI AND JHA: AN ANALYSIS OF LOGISTIC GROWTH MODEL WITH IMMIGRATION FUNCTION IN...

Kandel A. and Byatt W. J., 1978. Fuzzy sets, fuzzy algebra and fuzzy statistics. Proceeding of the IEEE, pp.1619-1639.

Kumar V. and Lal S., 2017. Stability analysis of logistic growth model with immigration effect. Indian Journal of Scientific Research, 16(1): 112-119.

Mondal S.P., Benerjee S. and Roy T.K., 2013. First order linear homogeneous ordinary differential equation in fuzzy environment. International Journal of Pure and Applied Sciences and Technology, 14(1): 16-26.

Mondal S.P. and Roy T.K., 2016. Solution of second order linear differential equation in fuzzy environment. Annals of Fuzzy Mathematics and Informatics, 11(2): 197-221.

Paul S., Mondal S.P. and Bhattacharya P., 2016. Discussion on fuzzy quota harvesting model in fuzzy environment: fuzzy differential equation approach.
Modeling Earth Systems and Environment, 70(2): $1-15$.

Plotnikov A.V. and Skripnik N.V., 2012. Fuzzy differential equations with generalized derivative. Journal of Fuzzy Set Valued Analysis, pp. 1-12.

Seikkala S., 1987. On fuzzy initial value problem. Fuzzy Sets and Systems, 24(3): 319-330.

Takata L., de Barros G.L.C. and Bede B., 2015. Fuzzy differential equations in various approaches. Springer International Publishing, AG Switzerland.

Tapaswini S. and Chakraverty S., 2013. Numerical solution of nth order fuzzy linear differential equations by homotopy perturbation method. International Journal of Computer Applications, 64(6): 5-10.

Zadeh L.A., 1965. Fuzzy sets. Information and Control, 8: $338-353$. 